\title{
Supervised Biometric System Using Multimodal Compression Scheme
}

\author{
Wafa Chaabane ${ }^{1}$, Régis Fournier ${ }^{1}$, Amine Naït-ali ${ }^{1,}{ }^{*}$, Julio Jacobo ${ }^{2}$, Marta Mejail ${ }^{2}$, \\ Marcelo Mottalli ${ }^{2}$, Heitor Ramos ${ }^{3}$, Alejandro C. Frery ${ }^{3}$, and Leonardo Viana ${ }^{3}$ \\ ${ }^{1}$ Université Paris-Est Créteil (UPEC), LiSSi. Biometrics Research Group, France \\ \{wafa.chaabane, rfournier, naitali\}@u-pec.fr \\ ${ }^{2}$ Universidad de Buenos Aires, Argentina \\ \{julio.jacobo, marta.mejail, mottalli\}gmail.com \\ ${ }^{3}$ Universidade Federal de Alagoas, Brazil \\ acfrery@gmail.com, lpviana@lccv.ufal.br
}

\begin{abstract}
This work is a part of a project supported by STIC Am-Sud where the main objective is to design an intelligent vision system to protect children from some critical information accessible from the Internet, from some videos or from some video games that are related to violence, wars, pornography, etc. Considered definitively not appropriate for their age, such multimedia contains can significantly offend young people. More specifically, in this paper, we are interested in discussing a general concept of a supervised biometric system that is controlled by specific tags embedded in video frames through a multimodal compression. Using a spiral insertion scheme, specific frequencies (TAGs) are compressed jointly with video frames in the region of insertion and then extracted for supervision purpose. The multimodal compression is considered here because it allows high-level robustness regarding the bitrates and downsampling.
\end{abstract}

Keywords: Face recognition, biometrics, Intelligent system, Lossy compression, Multimodal Compression.

\section{Introduction}

Nowadays, televisions and computers are more and more used in daily routine for both personal and professional purposes. Since, such devices are used permanently by various categories of people, including young and very young people. It happens hence that some multimedia contains, not appropriate, can significantly offend them (e.g. wars, violence, pornography, etc). Without control, dramatic consequences can occur.

The question that may arise is: how one can protect young people and children from such contains?

\footnotetext{
* Corresponding author.
} 
It is well known that some proposed systems require the use of passwords to protect the access to resources or require the installation of some specific software to filter illegal websites or those considered not appropriate for children. Generally, the process of filtering requires using IP addresses or some keywords, but the problem is that these solutions are known to be very limited and can be easily bypassed.

This work is a part of a project called BIOCHIP, organized between France, Argentina and Brazil and supported by STIC Am-SUD [1]. The purpose is to develop a system that allows identifying persons, estimating their ages and controlling any vision system (e.g. TV/computer) by filtering automatically, the multimedia information, based on the contains and the user age (see Fig.1). In other words, the system should define who is using the computer, watching the TV or playing the video game. Somehow, the purpose of this intelligent system is to allow efficient and safe interaction between the device and the user.
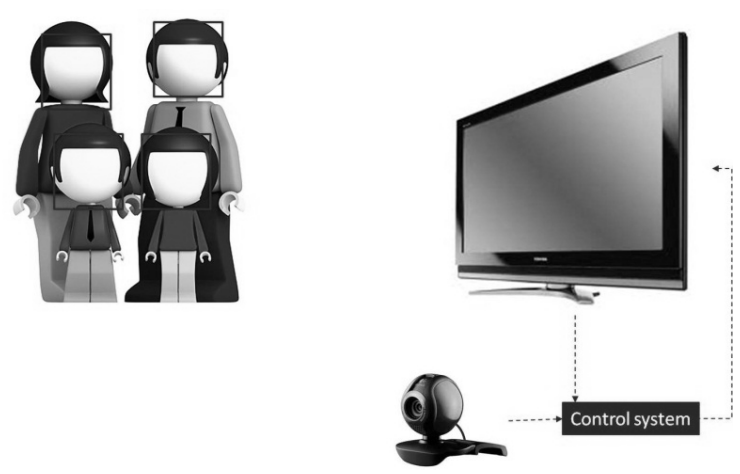

Fig. 1. Supervised intelligent TV system based on face recognition (images used separately from the Internet)

In this work, after describing briefly the concept, a special interest is given to the multimedia part that requires tagging the information (i.e. video) according to the category of its contains. For this purpose, no watermarking is used, but instead the video/images are compressed jointly with TAG, considered here as a signal having a specific frequency. This task is performed thanks to a multimodal compression scheme [4], [5] allowing a high robustness regarding the bitrates and which can be less sensitive to down-sampling.

This paper is organized as follows: in section 2, a general concept of face recognition module is presented. The multimodal compression scheme using a spiral mixing function is presented in section 3. Afterwards, some preliminary results are provided in section 4 , and finally a conclusion is given in the last section 5 .

\section{PCA-Based Identification}

Face detection and recognition are widely studied nowadays due to a requirement in identifying automatically persons [2]. They are used commonly for security and access control purposes. 
Face recognition systems do not require any physical contact since the image capture can be performed at a distance using a simple camera. In our system, principal component analysis (PCA) technique developed by Turk and Pentland [3] has been considered, after detecting faces.

This statistical technique requires the following processing steps:

1. The mean of the detected face images is removed,

2. Covariance matrix is calculated,

3. Eigenvectors (called also eigenfaces) are calculated from the covariance matrix,

4. Each face image is projected on the eigenfaces corresponding to the most significant eigenvalues. Consequently, each face image $k$ is characterized by a set of parameters $\left\{\alpha_{1 k}, \alpha_{2 k} \ldots \alpha_{M k}\right\}$.

The proposed system operates according a supervised mode. It required an enrolment phase and a control phase, described as follows:

1. Enrolment Phase: in this phase, the administrator should enrol the users that are supposed to watch the TV. A face recognition system extracts the parameters $\left\{\alpha_{1 k}, \alpha_{2 k} \ldots \alpha_{M k}\right\}$ from each acquired image using PCA method, as described above. Afterwards, the administrator attributes priorities for each user according to his age and the recommendation that should be given by the provider. For instance, if we consider the French legislation, one can attribute priorities according to the following categories:

-Category I: free program,

-Category II: not recommended for under 10 years old,

-Category III: not recommended for under 12 years old,

-Category IV: not recommended for under 16 years old,

-Category V: not recommended for under 18 years old.

Table 1. Matching face features and allowed priorities

\begin{tabular}{ccc}
\hline Person & Code & Priority \\
\hline 1 & $\left\{\alpha_{11}, \alpha_{21} \ldots \alpha_{M 1}\right\}$ & $\in\{I, I I, \ldots V\}$ \\
\hline 2 & $\left\{\alpha_{12}, \alpha_{22} \ldots \alpha_{M 2}\right\}$ & $\in\{I, I I, \ldots V\}$ \\
\hline$\ldots$ & $\ldots$ & \\
\hline $\mathrm{N}$ & $\left\{\alpha_{1 N}, \alpha_{2 N} \ldots \alpha_{M N}\right\}$ & $\in\{I, I I, \ldots V\}$ \\
\hline
\end{tabular}

Based on the same principle, other categories can be considered for video games that can differ from one country to another. Consequently, a priority $i$ is associated to a category $i$ (see Table 1).

2. Control Phase: during this phase, the system extracts tags from the analysed video frames and user face images in front the computer/TV are detected then identified in real time. Afterwards, a matching is performed between the identified category and the priority that corresponds to the youngest user (see Table 1, Fig. 2). 


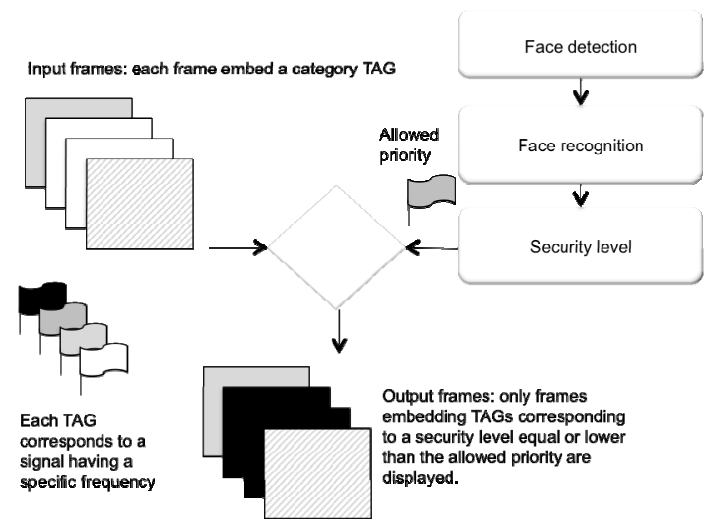

Fig. 2. Scheme showing the concept of biometric system supervision. Tags are embedded in input video frames before compression. Once decoded, only frames corresponding to a security level, equal or lower than the allowed priority are displayed.

\section{Multimodal Compression Tagging}

The idea of the multimodal compression consists in inserting "or embedding" signal samples into an image, before encoding the entire data mixture using only a single encoder [3]. As shown in a generic scheme of Fig. 3, the function allowing this mixture will be called "Mixing function". For the decoding, an inverse function, "Separation function" is applied to the decoded mixture. As evoked previously, in order to avoid any possible confusion, this technique shouldn't be considered as watermarking because the constraints and the purpose are not the same. Generally, when dealing with watermarking, one has to hide some data inside an image. This is not the case here.

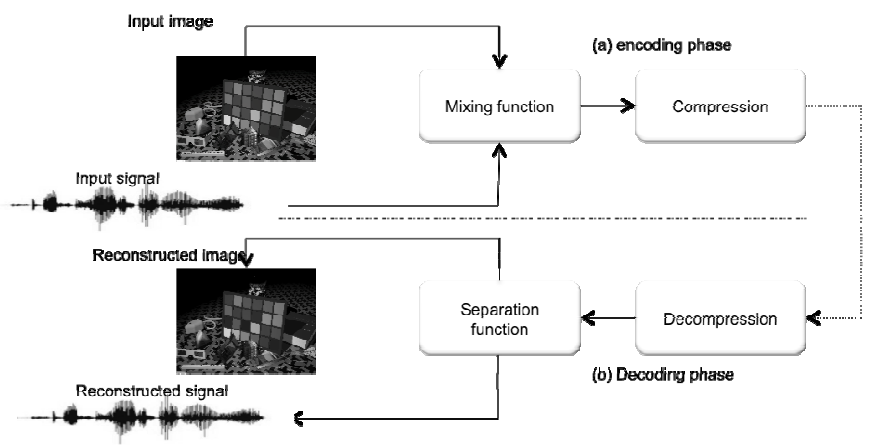

Fig. 3. Generic scheme of the multimodal compression showing a joint encoding image/signal

In our application, we have proposed a set of Tags where each Tag is a specific signal that corresponds to a given category. These Tags are embedded in the video frames according to a spiral mixing function, described bellow. These Tags correspond to five different frequencies $f_{1}, f_{2}, f_{3}, f_{4}, f_{5}$. When dealing with low compression ratios, it is indeed not necessary to insert these frequencies in each frame. 
Actually, when a frequency $f_{i}$ is detected, the priority $i$ is applied until the detection of another frequency. Whereas, when videos are compressed using high compression ratios, embedding Tags in each frame to create a redundancy is useful because, once extracted Tag signals can be averaged in order to reduce the noise due to the compression process (Fig. 6).

\subsection{Spiral Insertion Based Mixing Function}

Let's $I$ denotes a $M \times N$ frame and $s_{i}$ a $K$ samples Tag signal $f_{i}$. The insertion phase consists of replacing each sample/2 of a selected pathway that belongs to the frame, by Tag signal samples. In this work, we are considering a spiral pathway as shown in Fig. 4.

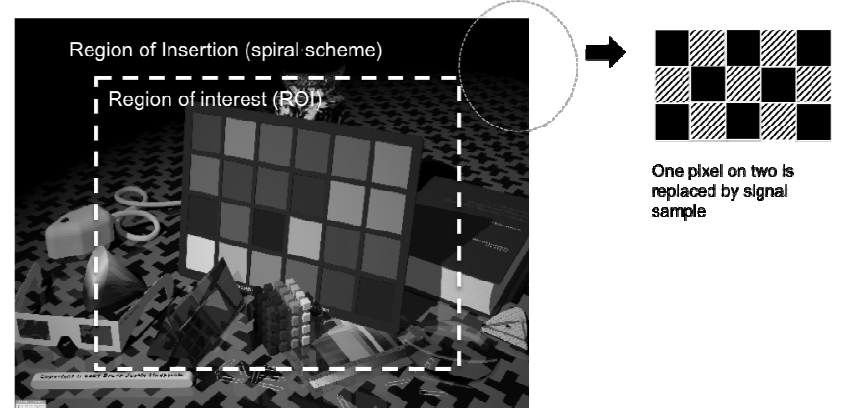

Fig. 4. Spatial insertion of samples in the Region of insertion. Tag signal samples are interleaved as shown (one pixel on two corresponds to Tag sample). No insertion is performed in $\mathrm{ROI}$ in order to avoid any potential distortion of the image quality.

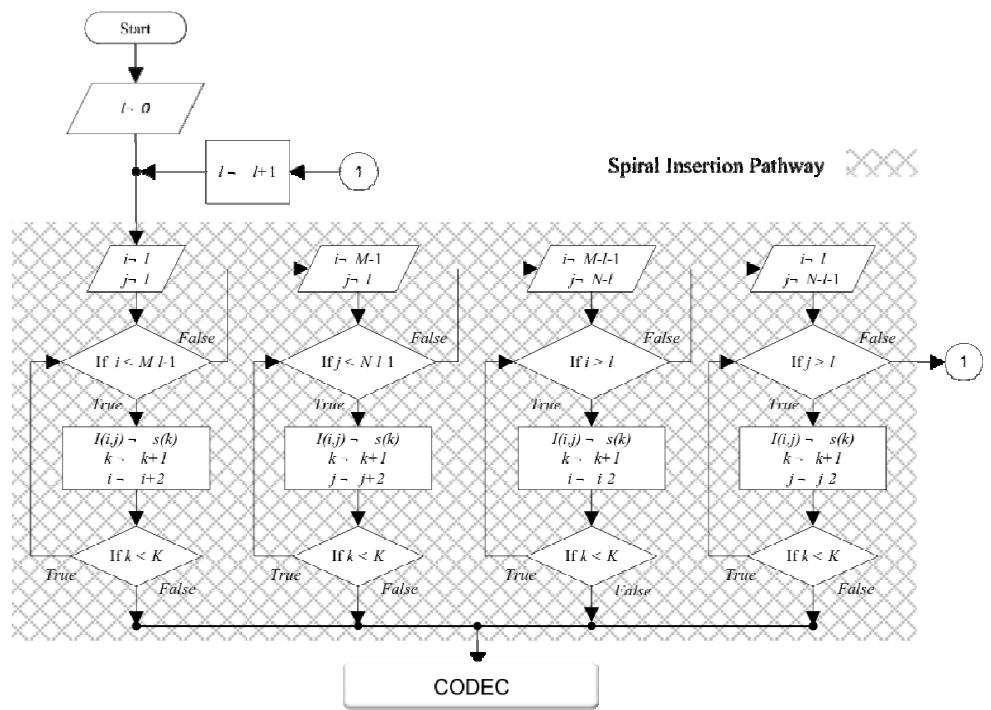

Fig. 5. Encoding flowchart corresponding multimodal compression scheme, using spiral insertion 
The insertion pathway should belong to the external insertion region (around the borders) because generally, Region of Interests (ROI) are basically located at the centre of the image. In such a case, the maximum of samples that can be inserted in an image without defining a ROI is $K \leq(M \times N) / 2$. This is referred as, the capacity of insertion. If a ROI is pre-defined in the frame (i.e. no insertion is allowed in that region), the capacity will be bounded by $((M-N)-S) / 2$ where $S$ denotes the size of the ROI.

The insertion algorithm is provided in the flowchart of the Fig. 5. Afterwards, the entire mixture is encoded.

\subsection{Spiral Separation}

At the decoding phase, the frame is first decoded. Afterwards, a separation function is achieved in order to separate the Tag signal from the frame. In this phase, signal samples are extracted from the spiral pathway to form the Tag signal (Figure 5). In order to reconstruct the original frame, pixels initially replaced by Tag signal samples should be estimated by a simple interpolation method.

In order to obtain high compression ratios, videos are generally compressed according to lossy compression standards (we used here the H.264 codec). Consequently, the quality of compressed images and the extracted Tags can be affected if the compression ratio is high. Since we present in this work only preliminary results, the PSNR (Peak Signal to Noise Ratio) function is used to evaluate the quality of the compressed images.

This PSNR should be evaluated in two regions, namely the global region denoted by PSNR $_{\text {global }}$ and the region where no Tag should be inserted ( $\left.\mathrm{PSNR}_{\text {local }}\right)$.

To evaluate the quality of the extracted Tag signal, one can use a time domain criterion such as the PRD (percent root-mean-square difference). In the frequency domain, the estimated frequency $\widehat{f}_{i}$ can be compared to the original one by a simple calculation of Euclidian distance (Fig. 6). In such a case, a threshold should be defined.

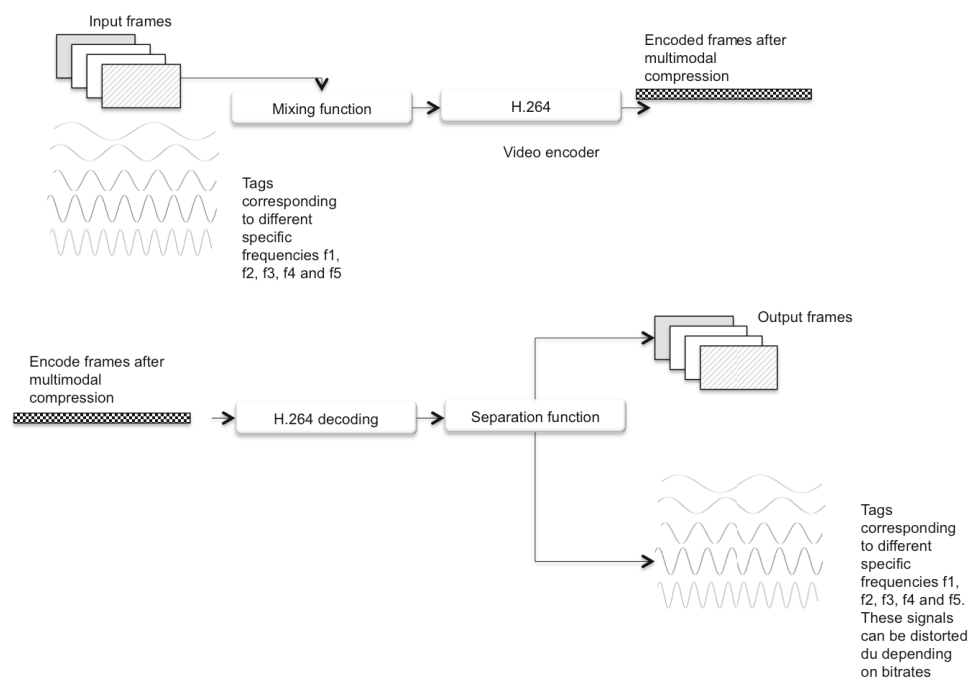

Fig. 6. Example of mixing/separation process using the multimodal compression scheme. Tags signals correspond to specific frequencies. 


\section{Some Preliminary Results}

To validate the proposed multimodal compression scheme, we have considered a speech signal (as a validation tool) before using Tag signals, since the speech signal contains a large number of frequencies. This signal is embedded into two different videos "Foreman" (Fig.7) and "Claire" using the spiral scheme mixing function. Afterwards, the mixture is compressed using H.264 codec. After the decoding process, the reconstructed frames as well as the reconstructed speech signal are evaluated in terms of quality. For example, Fig.8.(left), show that the quality of the decoded videos using H. 264 without multimodal compression is close to the quality obtained when considering multimodal compression. This means that for the same bitrate, the multimodal compression allows compressing an additional signal without affecting the quality of the video nor the quality of the embedded signal as shown in Fig 8.(right) Here, bitrate is set so that the visual quality of videos remains visually acceptable and without significant distortion.

Based on this result, Tag signals of the following frequencies are used $2000 \mathrm{~Hz}$, $2500 \mathrm{~Hz}, 3000 \mathrm{~Hz}, 3500 \mathrm{~Hz}$ and $4000 \mathrm{~Hz}$ that can be regarded as tones. The frequency sampling is set at $22050 \mathrm{~Hz}$. These signals are periodically embeded in each frame so that their extraction by averaging becomes possible even if the bitrate is low. As it has been reported previously, when dealing with high bitrates, it is not important to embed a Tag in each frame. Actually, a Tag signal should be embedded in a frame each time the video category changes. The same priority should be used until detecting a new Tag signal in the video.

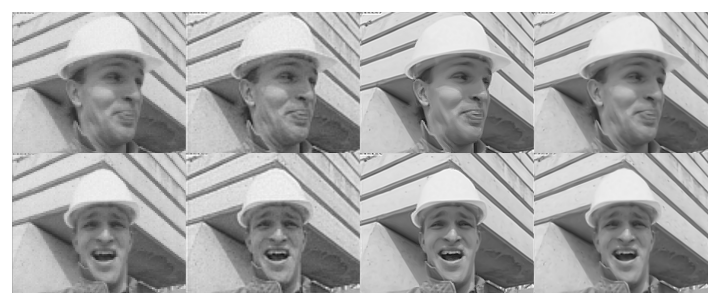

Fig. 7. Foreman video sequence used for the evaluation process

Consequently, using the multimodal compression based on a single encoder, an efficient encoding of both frames and the Tag signals are obtained.
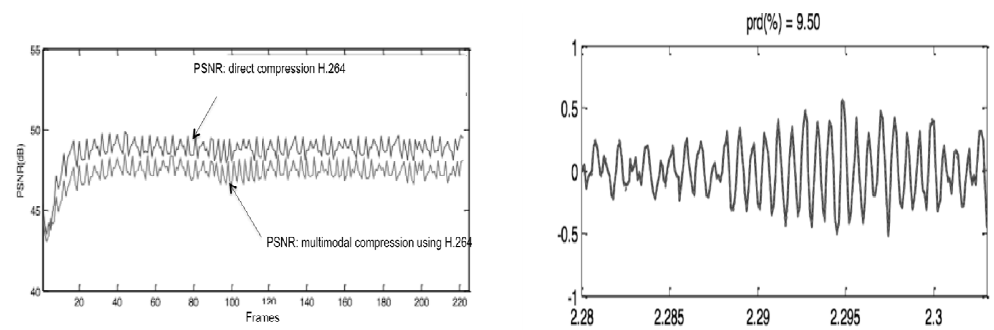

Fig. 8. Left: Mean video quality with and without embedding Tags. Right: Input embedded signal and the output superimposed ( $\mathrm{PRD}=9.50 \%)$, highlighting the minimal distorsion. 


\section{Conclusion}

We have considered in this paper a supervised vision system that is controlled through Tags embedded in video sequences and encoded using a multimodal compression scheme. The main advantage of this scheme is its robustness regarding the compression ratio. No significant distortion is observed on decoded videos, and good Tags quality is obtained whatever the used frequency, (validation achieved on speech signals). For a future work, it would be interesting to optimize both the mixing function and the shape of Tags in order to reduce the computing complexity.

\section{References}

1. BIOCHIP (2012), http://international-education-biometrics.net/biochip/

2. Zhao, W., Chellapa, R., Phillips, P.: Face recognition: a literature survey. ACM Computing Surveys 35(4), 399-458 (2003)

3. Turk, M., Pentland, A.: Eigenfaces For Recognition. Journal of Cognitive Neuroscience 3, 71-86 (1991)

4. Naït-ali, A., Zeybek, E., Drouot, X.: Introduction to Multimodal Compression of Biomedical data. book: Advanced Biosignal Processing, pp. 353-375. Springer (2009)

5. Fournier, R., Nait-ali, A., Zeybek, E.H.: A novel supervised model for multimodal compression. Journal of Signal and Information Processing (in press, 2012) 